nisms underlying their roles in kidney injury and repair are largely unknown.

Furthermore, ischemic injury, the focus of the Li et al. paper, may not have the same pathophysiologic pathways as nephrotoxic injury or sepsis. Another key step will be to understand why so many mechanistic studies in rodents have shown protection while clinical studies targeting the same pathways have failed. Does ischemic injury in native kidneys have a different pathophysiology than IRI in allografts when additional factors such as brain death, cold transport, and immunosuppressive treatment are superimposed? Finally, a recent surge of data has identified AKI as an important risk factor for CKD (21-23), and immune cells are likely important mediators between these two diseases, possibly related to autoimmunity (24). The type of immune cells involved in AKI-toCKD transition and the details of how they function will be important to elucidate.

\section{Acknowledgments}

This work was supported by grants from the NIH, the National Kidney Foundation, and the American Heart Association. Sanjeev Noel graciously assisted with the figure.

Address correspondence to: Hamid Rabb, Ross 965, The Johns Hopkins Hospital, 720 Rutland Ave., Baltimore, Maryland 21205, USA. Phone: 410.502.1556; Fax: 410.614.1643; E-mail: hrabb1@jhmi.edu.
1. Clarkson MR, Friedewald JJ, Eustace JA, Rabb H. Acute kidney injury. In: Brenner BM, ed. Brenner \& Rector's The Kidney. 8th ed. Philadelphia, Pennsylvania, USA: Saunders, Elsevier; 2008:943-986

2. Jang HR, Ko GJ, Wasowska BA, Rabb H. The interaction between ischemia-reperfusion and immune responses in the kidney. $J$ Mol Med. 2009;87(9):859-864.

3. Thornton MA, Winn R, Alpers CE, Zager RA. An evaluation of the neutrophil as a mediator of in vivo renal ischemic-reperfusion injury. Am J Pathol. 1989;135(3):509-515.

4. Rabb H, et al. Role of CD11a and CD11b in ischemic acute renal failure in rats. Am J Physiol. 1994;267(6 pt 2): F1052-F1058

5 . Kelly KJ, et al. Intercellular adhesion molecule1-deficient mice are protected against ischemic renal injury. J Clin Invest. 1996;97(4):1056-1063.

6. Burne MJ, et al. Identification of the CD4(+) T cell as a major pathogenic factor in ischemic acute renal failure. J Clin Invest. 2001;108(9):1283-1290.

7. Dong X, Swaminathan S, Bachman LA, Croatt AJ, Nath KA, Griffin MD. Resident dendritic cells are the predominant TNF-secreting cell in early renal ischemia-reperfusion injury. Kidney Int. 2007;71(7):619-628.

8. Zhai Y, Busuttil RW, Kupiec-Weglinski JW. Liver ischemia and reperfusion injury: new insights into mechanisms of innate-adaptive immune-mediated tissue inflammation. Am JTransplant. 2011;11(8):1563-1569.

9. Huang S, Apasov S, Koshiba M, Sitkovsky M. Role of A2a extracellular adenosine receptormediated signaling in adenosine-mediated inhibition of T-cell activation and expansion. Blood. 1997;90(4):1600-1610.

10. Li L, et al. Dendritic cells tolerized with adenosine $\mathrm{A}_{2 \mathrm{~A}} \mathrm{R}$ agonist attenuate acute kidney injury. J Clin Invest. 2012;122(11):3931-3942.

11. Deng J, et al. Star Interleukin-10 inhibits ischemic and cisplatin-induced acute renal injury. Kidney Int. 2001;60(6):2118-2128

12. Bonventre JV, Vaidya VS, Schmouder R, Feig P, Dieterle F. Next-generation biomarkers for detecting kidney toxicity. Nat Biotechnol. 2010;28(5):436-440.
13. Mishra J, et al. Neutrophil gelatinase-associated lipocalin (NGAL) as a biomarker for acute renal injury after cardiac surgery. Lancet. 2005; 365(9466):1231-1238

14. Molls RR, et al. Keratinocyte-derived chemokine is an early biomarker of ischemic acute kidney injury. Am J Physiol Renal Physiol. 2006;290(5):F1187-F1193.

15. Gandolfo MT, et al. Foxp3+ regulatory T cells participate in repair of ischemic acute kidney injury. Kidney Int. 2009;76(7):717-729.

16. Tögel F, Hu Z, Weiss K, Isaac J, Lange C, Westenfelder C. Administered mesenchymal stem cells protect against ischemic acute renal failure through differentiation-independent mechanisms. Am J Physiol Renal Physiol. 2005;289(1):F31-F42.

17. Humphreys BD, et al. Intrinsic epithelial cells repair the kidney after injury. Cell Stem Cell. 2008;2(3):284-291.

18. Huang Y, et al. Kidney-derived stromal cells modulate dendritic and T cell responses. J Am Soc Nephrol. 2009;20(4):831-841.

19. Grams ME, Rabb H. The distant organ effects of acute kidney injury. Kidney Int. 2012;81(10):942-948.

20. Yokota N, Burne-Taney M, Racusen L, Rabb H. Contrasting roles for STAT4 and STAT6 signal transduction pathways in murine renal ischemiareperfusion injury. Am J Physiol Renal Physiol. 2003;285(2):F319-F325.

21. Ishani $A$, et al. Acute kidney injury increases risk of ESRD among elderly. J Am Soc Nephrol. 2009;20(1):223-228.

22. Macedo E, Bouchard J, Mehta RL. Renal recovery following acute kidney injury. Curr Opin Crit Care. 2008;14(6):660-665.

23. Cohen SD, Kimmel PL. Long-term sequelae of acute kidney injury in the ICU [published online ahead of print August 29, 2012]. Curr Opin Crit Care. doi:10.1097/MCC.0b013e328358d3f5.

24. Burne-Taney MJ, Liu M, Ascon D, Molls RR, Racusen L, Rabb H. Transfer of lymphocytes from mice with renal ischemia can induce albuminuria in naive mice: a possible mechanism linking early injury and progressive renal disease? Am J Physiol Renal Physiol. 2006;291(5):F981-F986.

\title{
New mechanisms of glucocorticoid-induced insulin resistance: make no bones about it
}

\author{
Heather A. Ferris and C. Ronald Kahn \\ Joslin Diabetes Center, Harvard Medical School, Boston, Massachusetts, USA.
}

\begin{abstract}
Glucocorticoids are a powerful tool used to treat a range of human illnesses, including autoimmune diseases and cancer, and to prevent rejection following organ transplantation. While lifesaving for many, they come with a steep price, often leading to obesity, insulin resistance, diabetes, and osteoporosis. In this issue of the JCI, Brennan-Speranza and colleagues provide evidence that the osteoblast-derived peptide osteocalcin is one of the drivers of the metabolic derangements associated with glucocorticoid therapy. This novel mechanism could open up new avenues for the treatment of these disorders.
\end{abstract}

Conflict of interest: The authors have declared that no conflict of interest exists.

Citation for this article: J Clin Invest. 2012;

122(11):3854-3857. doi:10.1172/JCI66180.
Glucocorticoids act by binding to the glucocorticoid receptor and promoting binding of the receptor to glucocorticoid response elements or association with other transcription factors, such as AP1, leading to either transactivation or transrepression of target genes. At physiological concentrations, glucocorticoids have effects on multiple metabolic, cardiovascular, and immunologic functions. Pharmacologic concentrations produce their therapeutic benefit of immune modulation by inducing antiinflammatory cytokines, inhibiting inflammatory cytokines, and inducing apoptosis of T-lymphocytes (1) but also cause many undesirable side effects, including increased susceptibility to infection, weight gain, glucose intoler- 


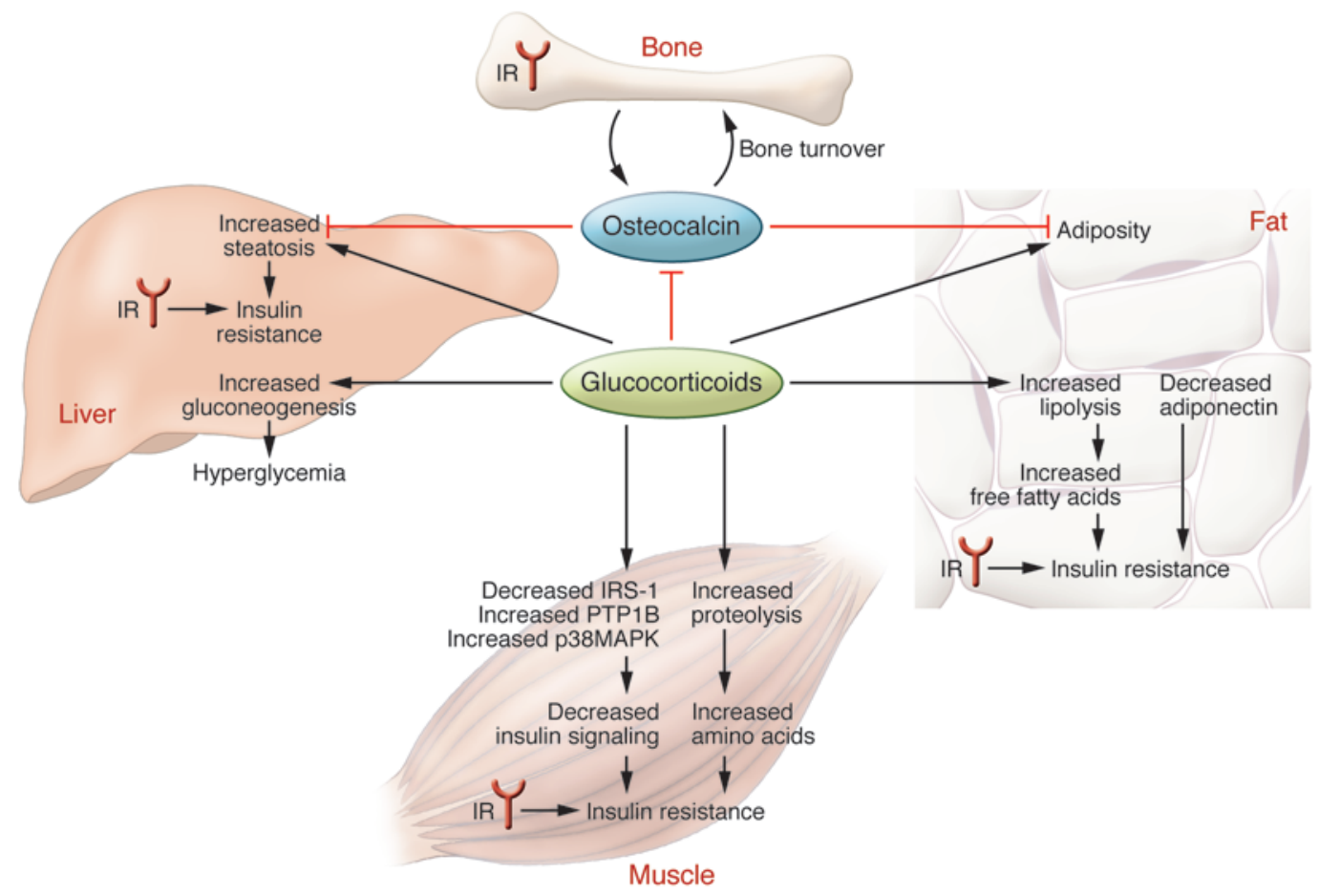

\section{Figure 1}

Mechanisms leading to glucocorticoid-mediated insulin resistance. Glucocorticoids exert their impact on metabolism through several different tissues in the body. In the presence of glucocorticoids there is an increase in adiposity as well as an increase in lipolysis, leading to elevated free fatty acids in the circulation and an increase in insulin resistance. Muscle undergoes proteolysis, releasing amino acids that increase insulin resistance. Postreceptor insulin signaling defects such as a decrease in IRS-1 also contribute to insulin resistance. In the liver, there is increased steatosis, causing insulin resistance, which is compounded by increased gluconeogenesis and hyperglycemia. The bone is the site of osteocalcin production, driven by the IR. Osteocalcin normally participates in bone turnover as well as suppresses increases in adiposity and steatosis. These actions are inhibited by glucocorticoids.

ance, increased skin fragility, muscle breakdown, negative calcium balance and osteoporosis, cataracts, and CNS effects.

\section{Glucocorticoids and insulin resistance}

One of the most distressing side effects for patients treated long term with glucocorticoids is weight gain, often with disfiguring fat deposition. In humans treated with glucocorticoids, the accumulation of adipocytes occurs primarily in the visceral fat and interscapular depots, leading to a characteristic "buffalo hump" and truncal obesity. This occurs through effects of glucocorticoids on differentiation of preadipocytes into mature adipocytes (2). Glucocorticoids also induce insulin resistance, hyperglycemia, and hyperlipidemia. While increases in visceral fat contribute to the insulin resistance that occurs with glucocorticoid therapy, direct actions of glucocorticoids on muscle, liver, and other tissues also play a role.

Glucocorticoids have been shown to inhibit a number of steps in the insulin signaling network through several differ- ent mechanisms (Figure 1). Insulin acts by binding to its receptor (IR), leading to increased kinase activity and tyrosine phosphorylation of several downstream signaling molecules, including IRS-1 through IRS-4. These proteins then activate the PI3K and MAPK pathways, leading to a range of downstream effects (3). In skeletal muscle, glucocorticoids cause insulin resistance by decreasing transcription of IRS-1, while increasing transcription of two proteins that counter insulin action, protein tyrosine phosphatase type 1B (PTP1B) and p38MAPK (4). A similar increase in transcription of p38MAPK is observed in liver (5). Glucocorticoids also decrease IRS-1 and IRS-2 levels in fat $(6,7)$, while there is a decrease in IR and IRS- 1 phosphorylation in response to glucocorticoids in liver $(6,8)$. In the 3T3-L1 mouse adipocyte cell line, there is upregulation of MAPK phosphatases, leading to downregulation of p38MAPK phosphorylation (8).

Factors secreted by adipose tissue, referred to as adipokines, also act to modify insulin sensitivity of tissues. One of these, adiponectin, promotes insulin sensitivity in tissues and is suppressed by glucocorticoid treatment, serving as yet another component in the insulin resistance seen with glucocorticoid therapy (9). Glucocorticoids also promote proteolysis, lipolysis, free fatty acid production, and fat accumulation in the liver, which can contribute to insulin resistance (9). In addition, glucocorticoids directly promote hepatic gluconeogenesis, increasing hyperglycemia. Pancreatic $\beta$ cell dysfunction allows further hyperglycemia to develop, likely contributing to the progression to frank diabetes sometimes seen with chronic glucocorticoid therapy (9).

In this issue of the JCI, Brennan-Speranza and colleagues provide evidence for a new mechanism of glucocorticoidinduced insulin resistance in which glucocorticoid action on bone decreases the levels of the osteoblast-derived peptide osteocalcin, which in turn contributes to the insulin resistance and metabolic derangements associated with glucocorticoid therapy (10). 


\section{Bone as a modulator of glucose homeostasis and insulin resistance}

Osteocalcin, or bone $\gamma$-carboxyglutamate (gla) protein (BGLAP), is a small, highly conserved protein made by osteoblasts that is secreted into the circulation and associates with the mineralized matrix of bone. Osteocalcin is also expressed at a low level in preadipocytes and has been shown to be secreted by human subcutaneous and omental adipose tissue in culture $(11,12)$. In 2007 , the Karsenty laboratory reported a surprising connection between bone and glucose homeostasis when they observed that osteocalcin knockout mice are glucose intolerant and insulin resistant (13). These mice also have larger fat pads and increased triglycerides. The proposed mechanisms for these abnormalities are a decrease in pancreatic $\beta$ cell mass, leading to hypoinsulinemia, and decreased adiponectin, causing insulin resistance. Another group demonstrated that disruption of the IR in osteoblasts was sufficient to reduce osteocalcin and produce similar metabolic changes (14). Follow-up studies have shown that osteocalcin infusion can reduce hyperphagia induced by hypothalamic toxicity and reduce high-fat diet-mediated obesity and insulin resistance, while increasing insulin secretion in response to a glucose challenge (15). Indeed, daily injections of osteocalcin in mice fed a high-fat diet result in decreased weight gain, decreased insulin resistance, reduced hepatic steatosis, and increased energy expenditure (16).

\section{Osteocalcin and the glucocorticoid problem}

The observation that glucocorticoids inhibit the transcriptional activation of osteocalcin through transrepression made decreases in osteocalcin a possible candidate for glucocorticoid-mediated bone loss (17). However, knockout of the osteocalcin gene in mice results in increased, not decreased, bone formation (18). In an attempt to address the contribution of osteoblasts to glucocorticoid-mediated bone loss, BrennanSperanza and colleagues used a transgenic mouse, Col2.3-11 $\beta$ HSD2, which selectively overexpresses $11 \beta$-hydroxysteroid dehydrogenase type 2 in osteoblasts and osteocytes (19). This results in accelerated breakdown of glucocorticoids in osteoblasts and osteocytes, without modulating glucocorticoid activity in the rest of the animal. While the bones of transgenic mice were partially protected from the adverse effects of systemic glucocorticoids, the more surprising phenotype was protection from the obesity, hyperlipidemia, and insulin resistance produced by glucocorticoids. Indeed, these transgenic mice are protected from the weight gain, increased triglycerides, and abnormal glucose metabolism associated with 4 weeks of treatment with corticosterone. This is accompanied by a partial rescue of osteocalcin levels (10).

In order to determine whether osteocalcin is the mediator of these osteoblastic effects, as previous data would suggest, these authors expressed osteocalcin in the livers of these mice in vivo. This recapitulated the results of previous experiments in the transgenic Col2.3-11ßHSD2 mice, significantly mitigating the metabolic side effects of glucocorticoid treatment. These data further strengthen the link between bone and metabolism and establish osteocalcin as a significant mediator of glucocorticoid-induced metabolic disruption.

\section{Strategies for preventing glucocorticoid-mediated insulin resistance}

Will osteocalcin make a move into the clinical realm for the treatment of obesity, diabetes, or glucocorticoid-induced alterations in bone and metabolism? There are certainly suggestions that it has clinical promise. By acting downstream of the glucocorticoid receptor, it appears that it may reverse much of the metabolic phenotype caused by glucocorticoids without interfering with the immunosuppressive effects. Additionally, in humans, serum osteocalcin levels in obese diabetics are lower than in nondiabetics with a similar BMI (20). Likewise, the uncarboxylated form of osteocalcin decreases with increasing obesity (11). The fact that osteocalcin can be given intermittently and achieve the same results as an infusion is also of huge practical significance (16). For these reasons, there may be a place for osteocalcin "replacement" therapy in diabetes and metabolic syndrome in the future. Since osteocalcin may increase insulin secretion (15), this could add to the beneficial effect but also increase the risk of hypoglycemia. However, how systemic osteocalcin therapy will impact bone metabolism is probably the greatest area for concern in the development of osteocalcin as a drug to treat insulin resistance. When glucocorticoid-induced osteocalcin suppression is partially rescued in osteoblasts, there is preserved bone structure but no improve- ment in bone formation rate; however, osteocalcin knockout mice have increased, not decreased, bone mass $(18,19)$. How osteocalcin affects bone metabolism in humans will have an enormous impact on the success of osteocalcin as a treatment for human disease.

Other strategies are already being explored to attempt to circumvent the side effects of glucocorticoids while maintaining the therapeutic benefits. One strategy for targeting endogenous glucocorticoidmediated insulin resistance is inhibition of $11 \beta$-hydroxysteroid dehydrogenase-1, which is responsible for converting cortisone to the more biologically active form cortisol. Preliminary studies of one such inhibitor have demonstrated positive effects in the treatment of type 2 diabetes in humans (21), but this needs to be validated in other, larger studies. Also, such an inhibitor would not be useful to block the adverse metabolic effects of pharmacologic glucocorticoid therapy, as it would also block the immunosuppressive effects of the drugs. Another approach is to develop selective glucocorticoid receptor modulators. These take advantage of the fact that many of the genes associated with side effects (e.g., osteocalcin; ref. 17) are modulated through glucocorticoid receptor binding to glucocorticoid response elements, whereas many of the therapeutic effects occur through interactions with glucocorticoid receptor-associated transcription factors, such as AP1 (22). A number of selective glucocorticoid receptor modulators have been studied (22), and at least one is currently undergoing a phase II clinical trial for the treatment of rheumatoid arthritis (NCT01393639). The results are still a few years off, but if this compound is able to suppress the immune system while preventing some of the adverse effects of glucocorticoids, it could mark a major advance for the treatment of glucocorticoid responsive disorders.

Address correspondence to: C. Ronald Kahn, Joslin Diabetes Center, One Joslin Place, Boston, Massachusetts 02215, USA. Phone: 617.309.2635; Fax: 617.309.2593; E-mail: c.ronald.kahn@joslin.harvard.edu.

1. Barnes PJ. Glucocorticosteroids: current and future directions. Br J Pharmacol. 2011;163(1):29-43.

2. Cristancho AG, Lazar MA. Forming functional fat: a growing understanding of adipocyte differentiation. Nat Rev Mol Cell Biol. 2011;12(11):722-734

3. Biddinger SB, Kahn CR. From mice to men: insights into the insulin resistance syndromes. Annu Rev Physiol. 2006;68:123-158. 
4. Almon RR, Dubois DC, Jin JY, Jusko WJ. Temporal profiling of the transcriptional basis for the development of corticosteroid-induced insulin resistance in rat muscle. J Endocrinol. 2005 184(1):219-232

5. Almon RR, DuBois DC, Jusko WJ. A microarray analysis of the temporal response of liver to methylprednisolone: a comparative analysis of two dosing regimens. Endocrinology. 2007;148(5):2209-2225.

6. Saad MJ, Folli F, Kahn JA, Kahn CR. Modulation of insulin receptor, insulin receptor substrate-1, and phosphatidylinositol 3-kinase in liver and muscle of dexamethasone-treated rats. J Clin Invest. 1993;92(4):2065-2072.

7. Buren J, Lai YC, Lundgren M, Eriksson JW, Jensen $\mathrm{J}$. Insulin action and signalling in fat and muscle from dexamethasone-treated rats. Arch Biochem Biophys. 2008;474(1):91-101.

8. Bazuine M, Carlotti F, Tafrechi RSJ, Hoeben RC, Maassen JA. Mitogen-activated protein kinase (MAPK) phosphatase- 1 and -4 attenuate p38 MAPK during dexamethasone-induced insulin resistance in 3T3-L1 adipocytes. Mol Endocrinol. 2004;18(7):1697-1707.

9. Mazziotti G, Gazzaruso C, Giustina A. Diabetes in Cushing syndrome: basic and clinical aspects. Trends Endocrinol Metab. 2011;22(12):499-506.

10. Brennan-Speranza TC, et al. Osteoblasts mediate the adverse effects of glucocorticoids on fuel metabolism. J Clin Invest. 2012;122(11):4172-4189.

11. Foresta $C$, et al. Evidence for osteocalcin production by adipose tissue and its role in human metabolism. J Clin Endocrinol Metab. 2010;95(7):3502-3506

12. Dorheim MA, et al. Osteoblastic gene expression during adipogenesis in hematopoietic supporting murine bone marrow stromal cells. J Cell Physiol. 1993;154(2):317-328.

13. Lee NK, et al. Endocrine regulation of energy metabolism by the skeleton. Cell. 2007;130(3):456-469

14. Fulzele K, et al. Insulin receptor signaling in osteoblasts regulates postnatal bone acquisition and body composition. Cell. 2010;142(2):309-319.

15. Ferron M, Hinoi E, Karsenty G, Ducy P. Osteocalcin differentially regulates beta cell and adipocyte gene expression and affects the development of metabolic diseases in wild-type mice. Proc Natl Acad Sci US A. 2008;105(13):5266-5270

16. Ferron M, McKee MD, Levine RL, Ducy P, Karsenty G. Intermittent injections of osteocalcin improve glucose metabolism and prevent type 2 diabetes in mice. Bone. 2012;50(2):568-575.

17. Heinrichs AA, et al. Identification of multiple glucocorticoid receptor binding sites in the rat osteocalcin gene promoter. Biochemistry. 1993; 32(42):11436-11444.

18. Ducy $\mathrm{P}$, et al. Increased bone formation in osteocalcin-deficient mice. Nature. 1996;382(6590):448-452.

19 . Henneicke $\mathrm{H}$, et al. Corticosterone selectively targets endo-cortical surfaces by an osteoblast-dependent mechanism. Bone. 2011;49(4):733-742.

20. Iglesias P, et al. Serum concentrations of osteocalcin, procollagen type $1 \mathrm{~N}$-terminal propeptide and beta-CrossLaps in obese subjects with varying degrees of glucose tolerance. Clin Endocrinol (Oxf). 2011;75(2):184-188

21. Rosenstock J, et al. The 11-beta-hydroxysteroid dehydrogenase type 1 inhibitor INCB13739 improves hyperglycemia in patients with type 2 diabetes inadequately controlled by metformin monotherapy. Diabetes Care. 2010;33(7):1516-1522.

22. De Bosscher K. Selective clucocorticoid receptor modulators. J Steroid Biochem Mol Biol. 2010; 120(2-3):96-104.

\section{Amazing IL-9: revealing a new function for an "old" cytokine}

\section{Edgar Schmitt and Tobias Bopp}

Institute for Immunology, University Medical Centre, Johannes Gutenberg University Mainz, Mainz, Germany.

\begin{abstract}
The incidence of melanoma - the most aggressive form of skin cancer - is dramatically increasing, while the development of innovative therapeutic strategies continues to be challenging, especially due to a lack of knowledge about the molecular mechanisms underlying melanoma progression as well as antitumor immunity. In this issue of the JCI, Yong Lu and colleagues report a central role for Th9 cells in antitumor immunity.
\end{abstract}

Pro-inflammatory cytokines have long been regarded as beneficial in robust antitumor immunity. Specifically, cytokines of the common cytokine receptor $\gamma$ chain family (such as IL-2, IL-15, and IL-21) have been tested for the treatment of melanoma and other tumors in humans (1). However, there is increasing evidence that depending on the individual tumor, inflammation can lead to either favorable or unfavorable clinical prognosis $(2,3)$. Recent clinical trials utilizing monoclonal antibodies blocking the inhibitory molecules programmed cell death 1 (PD-1) (4) or cytotoxic T lymphocyte-associated protein 4 (CTLA-4) (5) suggest that aggravation of an adaptive immune response is a promising therapeutic strategy for the treatment of melanoma.

Conflict of interest: The authors have declared that no conflict of interest exists.

Citation for this article: J Clin Invest. 2012; 122(11):3857-3859. doi:10.1172/JCI65929.
During the last two decades the laboratory of Qing Yi has significantly contributed to our current understanding of potential immunotherapies in human malignancies by describing the role of DCs - and particularly $\mathrm{T}$ cells - in multiple myeloma.

In this issue of JCI, Yong Lu and colleagues from the Yi laboratory elucidate the role of Th9 cells and the $\gamma$ chain family member IL-9 in a B16 melanoma mouse model, and found that Th9-derived IL-9 inhibited tumor progression (ref. 6 and Figure 1). In addition, Th9 cells induce IL-9-dependent expression of CCL20 in lung epithelial cells, thereby promoting the recruitment of $\mathrm{CD} 8 \alpha^{+} \mathrm{CCR} 6^{+} \mathrm{DCs}$, leading to a strong activation of tumorreactive $\mathrm{CCR}^{+} \mathrm{CD}^{+}$CTLs. Collectively, the authors demonstrate an IL-9-based antitumor potency of Th9 cells, which is in quality and quantity superior to the immune response steered by Th1 cells. Hereby, the authors corroborate similar data quite recently published by Purwar et al., showing that IL-9-producing Th9 cells substantially inhibit melanoma growth as well as lung carcinoma growth in a murine B16F10 model (7).

\section{IL-9 restrains tumor progression}

More than two decades ago, IL-9 was identified as p40 or TCGFIII, and it was functionally characterized as a growth factor for repetitively stimulated $\mathrm{T}$ cell lines, but not for naive $T$ cells (8). In addition to its effect on T cells, a mast cell growth-enhancing activity (MEA) was demonstrated, and p40/TCGFIII/MEA was renamed IL-9 (8). Subsequent research proved an essential role for IL-9 in tolerance induction and the pathophysiology of allergic asthma, inflammatory bowl disease, microbial infections, and autoimmunity (8).

In the present study, the authors demonstrate that neutralization of IL-9 in a B16 lung metastasis model resulted in strong tumor growth associated with a significant decrease in the number of $\mathrm{CD} 4^{+} \mathrm{T}$ cells, $\mathrm{CD}^{+} \mathrm{T}$ cells, CD8 $\alpha^{+} \mathrm{DCs}$, and CD $11 \mathrm{~b}^{+}$ DCs in the lung. Further analyses demonstrated that Th9 cell-derived IL-9 prevented tumor progression in prophylactic and therapeutic settings. Previous findings 